\title{
Reflections of executive staff using the SaferCare Victoria COVID-19 clinical screening tool in a residential aged care service during the pandemic in Victoria, Australia
}

\author{
AUTHORS \\ SONALI PINTO BN, MN(ClinLead $)^{1}$ \\ 1 Bupa, Victoria, Australia \\ ZOE WAINER BMBS, MPH, $\mathrm{PhD}^{1}$ \\ SUSAN OLIVER RN, GradDipCH ${ }^{1}$ \\ Melbourne, Victoria, Australia \\ JOSEPH E IBRAHIM PhD, FRACP, FAFPHM ${ }^{2}$

\section{CORRESPONDING AUTHOR} \\ JOSEPH E IBRAHIM School of Nursing and Midwifery, Monash University, 10 Chancellors Walk, \\ Clayton Campus, Victoria 3800 Australia. Email: joseph.ibrahim@monash.edu \\ orcid.org/0000-0003-2828-9160
}

2 School of Nursing and Midwifery, Monash University,

\section{ABSTRACT}

Screening tools are useful for identifying disease during an early and pre-symptomatic stage. Older people dwelling in residential aged care services are particularly susceptible to COVID-19 and if infected have a high mortality rate. This article describes the lived experiences and reflections of some of the executive staff of an aged care service following the use of a COVID-19 clinical screening tool developed by SaferCare Victoria. The reflections were based on experiences of the contributors to this article during the second wave of the pandemic during 2020 in Victoria, Australia.

Open learning sessions were held via teleconference for staff to be trained in use of the tool. The tool was used in a variety of different circumstances for monitoring all residents. At times residents would decline to have certain observations taken and clinical staff were initially concerned about the potential extra work.
The regular use of the tool to track a residents' clinical observations over a 14-day period allowed opportunities to identify early subtle changes from the individual's baseline. There was a perceived improvement in detection of residents with dehydration, delirium, urinary tract infections and those approaching the need for end-of-life care.

Using the tool appears to assist in shifting the aged care service to a more systematic approach to responding to the pandemic. This appears to benefit the organisation, facility, staff, residents and their families however, more empirical research is required to test and validate these perceptions.

Keywords: Aged care, COVID-19, nursing homes, screening 


\section{INTRODUCTION}

Screening tools are useful for identifying disease during an early and pre-symptomatic stage. ${ }^{1}$ This is especially important with infectious outbreaks such as COVID-19. Recognition and management of the person with early stages of COVID-19 may result in easier and less expensive treatment and reduces the risk of transmission of COVID-19 infection to others. ${ }^{2}$

Older people in residential aged care are particularly susceptible to COVID-19 and if infected have a high mortality rate. ${ }^{3}$ It is not practical to be using invasive, laboratory viral screening tests for the whole aged care sector on a daily basis. In contrast the use of a clinical screening tool for COVID-19 has the benefits of being non-invasive, low economic cost and simpler to apply. The SaferCare Victoria (SCV) clinical screening tool was designed and developed to identify older people in residential aged care who may have COVID-19 and to guide treatment including whether or not to conduct laboratory tests. 4,5

\section{OBJECTIVES}

This article describes our experiences as a self-selected group of executive staff employed at an approved aged care provider (SP, ZW, SO) using a screening tool in Victoria Australia during the second wave of the COVID-19 pandemic (Jul-Oct 2020). The provider operates 26 residential aged care facilities which accommodate approximately 2,500 residents in Victoria. The article is a reflection based on self-reported experiences of this executive group. The discussion was facilitated by the senior author (who is an independent academic with no relationship with the staff or provider) and does not use any other information about other persons, as such, institutional ethics committee approval was not required. There are many approaches to reflection and reflective practice. ${ }^{6}$ In essence reflection requires examining an event that has occurred, analyses of that event from a range of viewpoints and determining how the new insights apply to improve practice.

\section{PREPARING FOR A COVID-19 OUTBREAK}

In early 2020, the provider, had commenced reviewing policies, procedures, clinical protocols to align with guidance material available from Communicable Diseases Network Australia (CDNA), ${ }^{7}$ the Australian Health Protection Principal Committee (AHPPC), Commonwealth and State Health Departments. Also established were Daily Crisis Management Team Meetings (CMT) between clinical governance and operations teams. If a COVID outbreak was suspected Clinical Meetings were scheduled with the Clinical Care Managers (CCMs), Clinical Quality Consultants (CQCs) and the General Manager (GM).
It became clear when COVID-19 cases were increasing during June and August 2020 our organisation needed a clear clinical based agenda to define our initial approach to protecting residents, staff and families from COVID-19 outbreaks. Our impression was that the clinical teams at the individual residential aged care facilities were unprepared. Our experiences at the team meetings were that care staff struggled to identify and present details of the residents' clinical status in a comprehensive and systematic way.

We adopted the SCV Clinical Screening tool because it was designed using an evidence-based framework and could demonstrate when residents were displaying clinical deterioration that may indicate COVID-19. ${ }^{2}$ The format of the screening tool in the form of a single sheet per resident, however, was not useful as it created large volumes of paper which were difficult to review systematically and did not allow for ready assessment of individual resident trends nor observation of patterns within areas of the home. Prior to wider implementation we modified the format of the tool to allow every resident to be tracked and for a rapid analysis of information. This was achieved by using a spreadsheet over a 14-day period. We also added the clinical observations of blood pressure and oxygen saturation which were not part of the SCV Clinical Screening tool.

The clinical managers in each section or unit were tasked with populating the spreadsheet each shift. In order to streamline the process, the form had as many fields as possible pre-populated and Registered Nurses (RNs) were required to complete clinical observations each shift.

\section{USE OF THE SCV CLINICAL SCREENING TOOL}

We used the tool in a variety of different circumstances including monitoring of all residents located in a nursing home where:

- a confirmed COVID-19 outbreak, and response has been initiated (community transmission has occurred)

- a precautionary outbreak response has been initiated

- the community was defined as a hotspot or Local Government Area (LGA) under Level 4 restrictions

The tool was also used for residents who were:

- a new admission

- returning to the home from an outing

- returning to the home after hospitalisation with COVID-19 and were deemed no longer infectious (residents in COVID-19 Recovery Wards)

Residents were screened at least twice a day and up to four times a day if they were flagged as being at risk. 


\section{HOW WE TRAINED STAFF TO USE THE SCV CLINICAL SCREENING TOOL}

Open learning sessions were held via teleconference for RNs, Clinical Care Managers (CCMs) and Clinical Quality Consultants. We repeated these sessions as well as providing examples of a completed tool for staff to refer to. The excel spreadsheet we developed also had instruction prompts on how to complete the tool. Verbal instructions on how to use the clinical screening tool were discussed with the CCMs each morning at the daily clinical meetings including the need for extra monitoring, referral and escalation to Medical Practitioners or other specialists should this be deemed necessary.

\section{HOW WE MANAGED STAFF AND RESIDENT CONCERNS}

At times residents would refuse to have certain observations taken, however most of the time residents were understanding of the need and complied with staff requests.

Clinical staff were initially concerned about the potential extra work using the screening tool would entail. However, this concern was readily overcome as staff numbers were often increased and cohorted which enabled them to assess residents and have their data ready for consideration at daily clinical meetings. These clinical meetings were a chance for staff and management to discuss any residents who may be deteriorating and highlight those who required referral or escalation of their care. Clinical teams became appreciative of the daily clinical meetings often commenting that it assisted with initiating hospitalisation and referral as the recommendations were cited to have been made by the Clinical Review Team for individual residents.

\section{FEEDBACK FROM OUR STAFF WHEN USING THE TOOL}

Staff provided feedback that a change in temperature was often the first indicator. This was typically a low grade increase overnight from the residents' baseline. Staff flagged any residents with a temperature of $>37^{\circ} \mathrm{C}$ for closer clinical monitoring. Those who later tested positive for COVID-19 usually had a temperature rise to $37.4^{\circ} \mathrm{C}$ and then perhaps to above $38{ }^{\circ} \mathrm{C}$. A higher pulse rate was often observed, usually about 20-3o beats per minute above the resident's baseline while other residents had a drop in their oxygen saturation levels.

The symptoms of COVID-19 we observed more frequently in our residents who later tested positive to COVID-19 were lethargy, a low-grade increase in temperature, an increased heart rate and a drop in oxygen saturation.
Far less common was a change in a resident's sense of taste or loss of smell. Interestingly, falls were mostly seen during the post COVID-19 recovery period due to physical deconditioning.

\section{GREATEST VALUE OF USING THE TOOL}

The regular use of the tool to track a residents' clinical observations over a 14-day period allowed opportunities to identify early subtle changes from the individual's baseline. This led to early detection of changes that may be due to COVID-19, facilitated early testing and provided information to inform decisions about need for hospitalisation. The documentation of serial clinical observations and changes in presentation also provided objective evidence that convinced ambulance paramedics and acute hospital staff of the need to transfer a resident to hospital for acute management of their condition.

An additional benefit of the screening tool was the early detection of clinical deterioration in residents from conditions other than COVID-19. We also believe this improved our detection of dehydration, delirium, urinary tract infections and those approaching the need for end-oflife care.

\section{THE OVERALL PERCEIVED BENEFITS OF USING THE SCREENING TOOL}

For the organisation:

- confirmation of an evidence-based process and a consistently reliable clinical process.

For the facility:

- assisted with decision making with cohorting and to predict infection control risks in transmission e.g., possibility of a positive cohort staff member etc.

- helped prioritise care and escalation of unwell residents.

For staff:

- perception that the exercise supported those RNs involved in the daily review process and their recognition of clinical deterioration improved.

For the resident:

- potentially a quicker hospitalisation and timely treatment.

For families of residents:

- perception that families felt reassured that there was a planned response to clinical monitoring and management. Families were appreciative their loved ones were being closely monitored and that appropriate treatment options were sought. 


\section{LIMITATIONS OF REFLECTION}

The observations reported in this article are the perceptions of the contributors. There is the potential for recall and social desirability bias which may lead to a more favourable evaluation of the screening tool. Objective, prospective empirical data at the individual staff, resident and family level is required to verify these perceptions.

\section{CONCLUSION}

By using the SCV screening tool we believe our approach to the pandemic was more systematic and our overall impression is that the tool provided a structure that assisted our staff to identify residents at risk and initiated earlier actions.

\section{DECLARATIONS}

All authors meet the criteria for authorship stated in the Uniform Requirements for Manuscripts Submitted to Biomedical Journals and are in full agreement regarding its content. This material has not been previously published and is not under consideration for any other publication. If accepted the manuscript will not be published elsewhere. One author is affiliated and employed by the School of Nursing, Monash University, which is also a funding source. The authors have no other potential financial or personal interests that may constitute a source of bias. The funding organisation did not contribute to, nor influence the content of the manuscript.

\section{DISCLAIMERS}

The views and opinions expressed in this article are those of the authors and do not necessarily reflect the official policy or position of any agency or departments of the Australian Federal Government, the State Government of Victoria, Bupa, Monash University, the Victorian Institute of Forensic Medicine or the Coroners Court of Victoria.

\section{REFERENCES}

1. Wilson JMG, Jungner G. Public health papers number 34: principles and practice of screening for disease. World Health Organization. Geneva. 1968. [cited 2021 Oct] Available at: http://apps.who.int/iris/bitstream/handle/10665/37650/ WHO PHP 34.pdf? sequence $=17$

2. Luo Z, Ang MJY, Chan SY, Yi Z, Goh YY, Shuangqian Y, et al (2020). Combating the coronavirus pandemic: early detection, medical treatment, and a concerted effort by the global community. Research. 2020; 2020:6925296. Available from: https://doi.org/10.34133/2020/6925296

3. Comas-Herrera A, Zalakaín J, Litwin C, Hsu AT, Lemmon E, Henderson D, et al. Mortality associated with COVID-19 outbreaks in care homes: early international evidence. International Long Term Care Policy Network. 2020. [cited 2021 Oct] Available from: https://Itccovid.org/wp-content/ uploads/2020/06/Mortality-associated-with-COVID-amongpeople-who-use-long-term-care-26-June-1.pdf

4. Safer Care Victoria. COVID-19 screening tool for residential aged care services. Safer Care Victoria. [cited 2021 Oct] Available from: https://www.bettersafercare.vic.gov.au/clinicalguidance/older-people/covid-19-screening-tool-for-residentialaged-care-services

5. Gnanasambantham K, Aitken G, Morris B, Simionato J, Chua EH, Ibrahim JE. Developing a clinical screening tool for identifying COVID-19 infection in older people dwelling in residential aged care services. Australas J Ageing. 2021;40(1):48-57. Available from: https://doi.org/10.1111/ajag.12884

6. Mann K, Gordon J, MacLeod A. Reflection and reflective practice in health professions education: a systematic review. Adv in Health Sci Ed 2009;14:595-621. Available from: https://doi.org/10.1007/s10459-007-9090-2

7. Department of Health. Coronavirus Disease 2019 (COVID-19) outbreaks in residential care facilities: CDNA National Guidelines for the prevention, control and public health management of COVID-19 outbreaks in residential care facilities in Australia. Australian Government. Canberra. 2020. [cited 2020 Apr] Available from: https://www.health.gov.au/sites/ default/files/documents/2020/05/coronavirus-covid-19guidelines-for-outbreaks-in-residential-care-facilities.pdf 